\title{
Random Early Discard (RED-AQM) Performance Analysis in Terms of TCP Variants and Network Parameters: Instability in High-Bandwidth-Delay Network
}

\author{
Mohammad Abu Obaida \\ Dept. of CSE, DUET \\ Gazipur-1700, \\ Bangladesh
}

\author{
Md. Sanaullah Miah \\ Dept. of CSE, DUET \\ Gazipur-1700, \\ Bangladesh
}

\author{
Md. Abu Horaira \\ Software Engineer \\ DataSoft BD Limited \\ Chittagong, Bangladesh
}

\begin{abstract}
Conventional congestion control methods (e.g. DROP TAIL) discards all received packets after the queue is full moreover results in low-network performance. To address this problem, RED was proposed to improve the performance of TCP connections. As a queue management mechanism, it drops packets in the considered router buffer to adjust the network traffic behavior according to the queue size. In application, TCP Variants (Reno, NewReno, Vegas, Fack and Sack1) show oscillatory curve of packet reception if RED is considered for queuing, besides, some variants out performs in receiving packets over different network parameters that this paper analyzes and finds out. However, an increase in link capacity (with the resulting increase of per-flow bandwidth) will cause significant degradation in TCP's performance, irrespective of the queuing scheme used. Hence the network is prone to instability with the rise in the number of High-bandwidth-delay product that is also attended to in this paper.
\end{abstract}

\section{General Terms}

Network Congestion Control, TCP Variants, Network Parameters, Queuing, Drop Tail, High-bandwidth-delay and Random Early Marking.

\section{Keywords}

RED, AQM, BW, TCP Variants, NS-2.

\section{INTRODUCTION}

Random Early Marking puts forward a great deal of improvement for the purpose of Congestion Control rather than conventional Drop Tail mechanism does. The rationale behind the discrepancy in RED performance for different TCP Variants is that each of the Variants type possesses some special decisive factors. Such as, the base TCP has become known as TCP Tahoe. TCP Reno attaches one novel mechanism called Fast Recovery to TCP Tahoe [6]. In addition, TCP Newreno employs the most recent retransmission mechanism of TCP Reno. [7]. The use of Sacks allows the receiver to stipulate several additional data packets that have been received out-of-order within one dupack, instead of only the last in order packet received [8]. TCP Vegas offers its own distinctive retransmission and congestion control strategies. TCP Fack is Reno TCP with forward acknowledgment [9]. Transmission Control Protocol (TCP) Variants Reno, NewReno, Vegas, Fack and Sack1 are implemented in NS-2. RED supervises the average queue size and drops packets based on statistical likelihoods [2].

\section{RANDOM EARLY MARKING}

\subsection{RED, an improvement over Drop Tail}

Droptail network components discard packets when its FIFO queue is full. Under heavy load conditions, droptail routers grounds global synchronization, a phenomenon in which all senders sharing the same bottleneck router/link shut down their transmission windows at almost the same time, thereby causing a abrupt drop in the bottleneck link exploitation [10]. Besides, droptail routers are biased against bursty sources [11]. This is because, when a burst of packets from a sender arrives at a fully occupied queue, a sustained packet drop within the same window of data occurs. Floyd et al. [12] and Xu and Ansari [13] showed that TCP [14], lacks the capability to recuperate from such multiple packet losses within the same window of data. Therefore, the TCP sender has to rely on retransmission timeouts to recover the lost packets that considerably slows down the transmission rate of a TCP flow. On the Contrary, RED monitors the average queue size and drops packets based on statistical probabilities [2]. As the queue grows, the probability for dropping an incoming packet grows too. RED is shown to effectively tackle both the global synchronization problem and the problem of bias against bursty sources [1].

\subsection{RED Parameter Settings}

Average queue size $a v g$ is formulated [1] as:

$$
a v g \leftarrow(1-w q) \times a v g+w_{q} \times q
$$

Where, $w_{q}$ is the queue weight, $q$ is current queue size.

$w_{q}$ should have lower value for burstier traffic; more weight

is given in this case for the historic size of the queue.

As avg varies from $\min _{t h}$ to $\max _{t h}$, the packet-marking probability $p_{b}$ varies linearly from 0 to $\max _{p}$.

$$
p_{b} \leftarrow \frac{\max _{p} \times\left(\operatorname{avg}-\min _{t h}\right)}{\max _{t h}-\min _{t h}}
$$


The final packet-marking probability $p_{a}$ increases slowly as the count increases since the last marked packet [1]:

$$
p_{a} \leftarrow \frac{p_{b}}{1-\operatorname{count} \times p_{b}}
$$

\section{To implement RED with NS-2 or other simulation software:}

The optimal setting for $\min _{t h}$ (minimum threshold) depends on exactly what the desired tradeoff is at that router between low average delay and high link utilization. The burstier the traffic arrival process, the higher $\min _{t h}$ would have to be to achieve a given average link utilization.

1. NS-2 simulator allows basic RED automatic setting of parameters [8] according the expected RTT. By setting RED's parameters (thres $=0$, maxthres $=0$, q_weight $=-1$ ), this mechanism can be enabled.

2. The popular RED rule of thumb that is published in Sally Floyd's discussion on RED parameters [15]. The parameters are $\min _{t h}=5$ (ascertained or assumed), $\max _{t h}=3 \times \min _{t h}, \max _{p}=0.1$, $w_{q}=0.002$. The rules, of course, are flexible, e.g. burstier communication should have lower $w_{q}$.

3. Using fluid-flow model of TCP behavior to predict and achieve stability of the linear control system, model for setting RED parameters [3] is given by-

$$
\frac{\max _{p}}{\max _{t h}-\min _{t h}}=L_{r e d} \leq \frac{\left(2 N^{-}\right)^{2}}{\left(R^{+} C\right)^{3}} \times \sqrt{\frac{w_{g}^{2}}{K^{2}}+1}
$$

Where:

$$
K=\frac{\log _{e}(1-\alpha)}{\delta}
$$

When the number of nodes: $N \geq N$.

Average RTT:

$$
R_{0} \leftarrow \frac{q_{0}}{C}+T_{p}
$$

Maximum expected RTT: $\mathrm{R}_{0} \leq \mathrm{R}^{+}$

$$
w_{g}=0.1 \times \operatorname{minimum}\left\{\frac{2 N^{-}}{\left(R^{+}\right)^{2}} \times C, \frac{1}{R^{+}}\right\}
$$

$\left(w_{g}^{-1}\right.$ is approximately the time constant of the feedback loop).

$L_{\text {red }}$ should have the highest possible value; $K$ is usually set to 0.005 .

$T_{p}=$ Propagation Delays $(\mathrm{sec})$

$C=$ Line Capacity (packets/seconds)

\section{PERFORMANCE ANALYSIS}

\subsection{Variation in Bandwidth over Simulation}

Red queuing in application with respect to various TCP variants, in a condition of varied bandwidth maintaining other parameters unaltered, number of packets received are mentioned in Table 1 (90 sec simulation), Table 2 (240 sec simulation) and packets received vs. bandwidth is delineated consequently in Figure 1, Figure2. As illustrated in the graph, it is apparent that some variants perform very sound under low bandwidth conditions, however, other shows steady or improved performance for a range of bandwidths.

Table 1. Total number of packet received for various TCP variants with respect to Bandwidth for simulation time $90 \mathrm{~s}$.

\begin{tabular}{|c|c|c|c|c|c|}
\hline TCP Variants & \multicolumn{5}{|c|}{ Bandwidth } \\
\hline & $2 \mathrm{Mb}$ & $4 \mathrm{Mb}$ & $8 \mathrm{Mb}$ & $16 \mathrm{Mb}$ & $32 \mathrm{Mb}$ \\
\hline Reno & 2264 & 2050 & 1986 & 2000 & 1916 \\
\hline NewReno & 2303 & 1944 & 2246 & 2048 & 2013 \\
\hline Vegas & 1833 & 1738 & 2098 & 1573 & 2011 \\
\hline Fack & 1938 & 1963 & 2187 & 2222 & 2166 \\
\hline Sack1 & 2314 & 1963 & 1849 & 2175 & 1989 \\
\hline
\end{tabular}

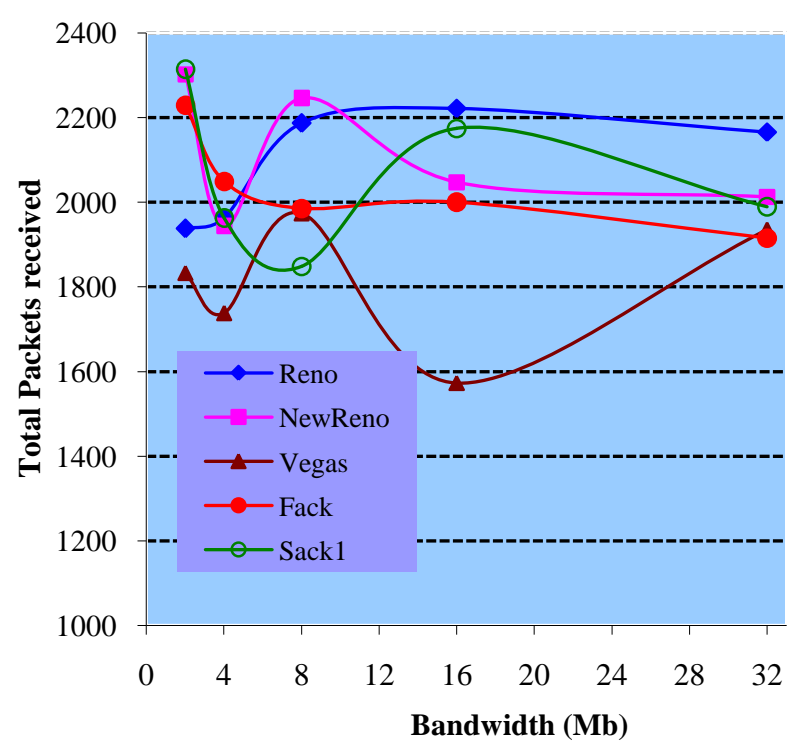

Figure 1.Graph of total packets received for various TCP Variants with respect to Bandwidth for simulation of 90s.

Table 2. Total number of packet received for various TCP variants with respect to Bandwidth for simulation of $240 \mathrm{~s}$

\begin{tabular}{|c|c|c|c|c|c|}
\hline TCP Variants & \multicolumn{5}{|c|}{ Bandwidth } \\
\hline & $2 \mathrm{Mb}$ & $4 \mathrm{Mb}$ & $8 \mathrm{Mb}$ & $16 \mathrm{Mb}$ & $32 \mathrm{Mb}$ \\
\hline Reno & 5889 & 5234 & 5476 & 5975 & 5648 \\
\hline NewReno & 6012 & 5469 & 5349 & 5895 & 5648 \\
\hline Vegas & 4774 & 4833 & 4972 & 4757 & 5249 \\
\hline Fack & 5355 & 5663 & 5605 & 5603 & 5836 \\
\hline Sack1 & 5782 & 5077 & 5624 & 5603 & 5170 \\
\hline
\end{tabular}




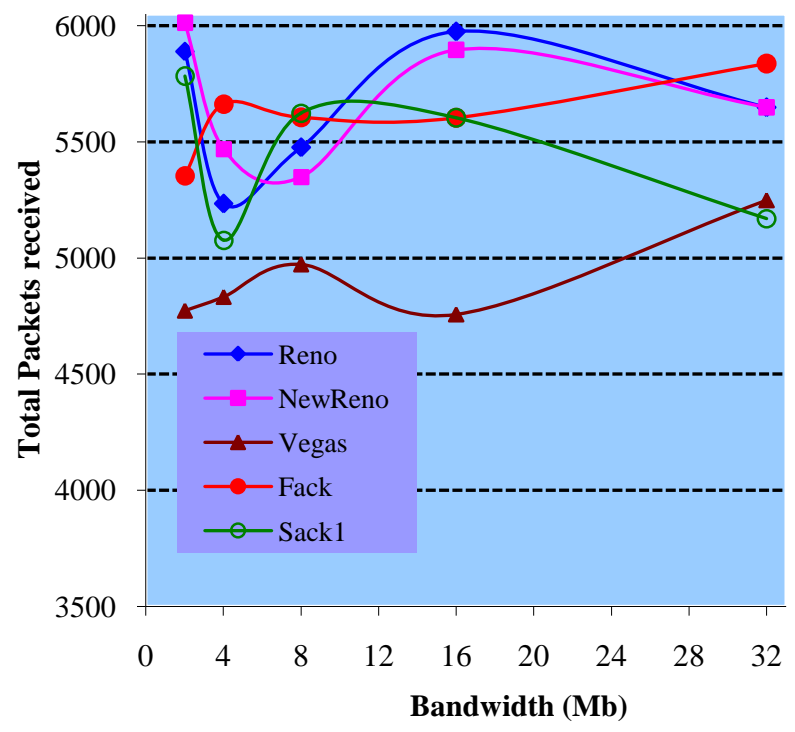

Figure 2.Graph of total packets received for various TCP Variants with respect to Bandwidth for 240s simulation.

\subsection{Variation in Delay over Simulation}

Some of the TCP Variants are indifferent to the variation in delay; on the contrary, other variant seems extremely sensitive to the alteration in the delay. Red queuing in application with respect to various TCP variants number of packets received are mentioned in Table 3 (90sec simulation), Table 4 (240sec simulation) and depicted accordingly in Figure 3, Figure 4. As demonstrated in the graph, easily noticed that some variants operates exceptionally well under low bandwidth settings, however, other shows stable or improved performance for a band of bandwidths.

Table 3. Total number of packet received for various TCP variants with respect to Delay for simulation of $90 \mathrm{~s}$.

\begin{tabular}{|c|c|c|c|c|c|c|}
\hline TCP Variants & \multicolumn{6}{|c|}{ Delay } \\
\hline & $1 \mathrm{~ms}$ & $5 \mathrm{~ms}$ & $10 \mathrm{~ms}$ & $20 \mathrm{~ms}$ & $50 \mathrm{~ms}$ & $100 \mathrm{~ms}$ \\
\hline Reno & 2326 & 2106 & 2230 & 1888 & 2133 & 1967 \\
\hline NewReno & 1838 & 1976 & 2303 & 2356 & 2134 & 2120 \\
\hline Vegas & 1860 & 1770 & 1833 & 1990 & 1889 & 2089 \\
\hline Fack & 1997 & 1767 & 1938 & 2023 & 1970 & 2108 \\
\hline Sack1 & 2029 & 1949 & 2314 & 1860 & 1947 & 1813 \\
\hline
\end{tabular}

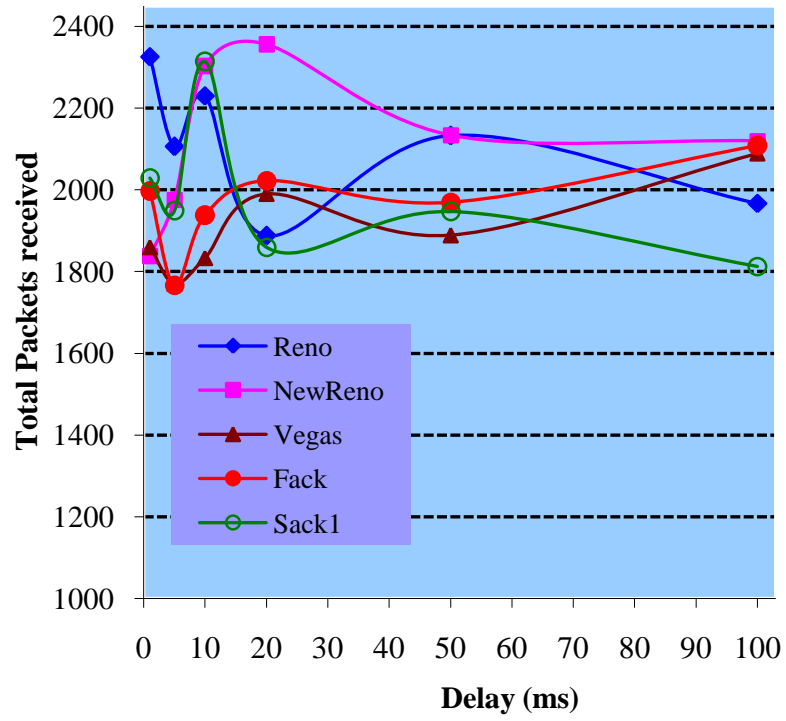

Figure 3.Graph of total packets received for various TCP Variants with respect to Delay for simulation of $90 \mathrm{~s}$.

Table 4. Total number of packet received for various TCP variants with respect to Delay for simulation of 240s.

\begin{tabular}{|c|c|c|c|c|c|c|}
\hline TCP Variants & \multicolumn{6}{|c|}{ Delay } \\
\hline & $1 \mathrm{~ms}$ & $5 \mathrm{~ms}$ & $10 \mathrm{~ms}$ & $20 \mathrm{~ms}$ & $50 \mathrm{~ms}$ & $100 \mathrm{~ms}$ \\
\hline Reno & 5702 & 5329 & 5889 & 5056 & 5456 & 5636 \\
\hline NewReno & 5700 & 5536 & 6012 & 5987 & 5170 & 5381 \\
\hline Vegas & 4763 & 4849 & 4774 & 4855 & 4554 & 4894 \\
\hline Fack & 5984 & 5595 & 5355 & 5954 & 5241 & 5407 \\
\hline Sack1 & 5607 & 5664 & 5782 & 5775 & 5561 & 5580 \\
\hline
\end{tabular}

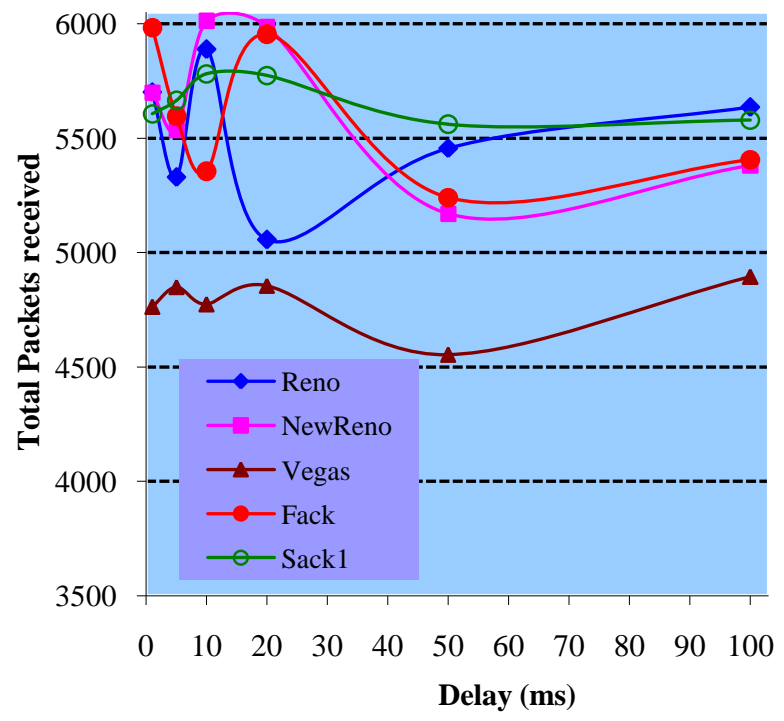

Figure 4.Graph of total packets received for various TCP Variants with respect to Delay for simulation of 240s. 


\subsection{Rating the TCP Variants}

To deduce, a deliberate scrutiny of the statistics and graphs demonstrated above advocate the following findings and decisions for RED queuing-

Variation in Bandwidth over Simulation (3.1):

Reno, NewReno, Fack $\rightarrow$ Receive more packets and steady. NewReno, Sack1, Reno $\rightarrow$ Excellent performance in low BW. Vegas, Sack1, Newreno $\rightarrow$ Unstable over variation in BW. =>Most consistent and better performance literally: Reno.

Variation in Delay over Simulation (3.2):

NewReno, Reno, Sack $1 \rightarrow$ Receive more packets.

Reno, Fack, Sackl $\rightarrow$ Superior performance in low Delay.

Vegas, Sack $1 \rightarrow$ Unstable over variation in delay.

Sackl, Vegas, Fack $\rightarrow$ Least delay sensitive.

=>Most regular and better performance literally: NewReno.

\subsection{Responses in High-Bandwidth-Delay Network:}

Irrespective of the queuing scheme used, an increase in link capacity (with the ensuing increase of per-flow bandwidth) will cause considerable degradation in TCP's performance [5].By casting the problem into a control theory framework, Low et al. [16] show that as capacity or delay increases, Random Early Discard (RED) [1], Random Early Marking (REM) [17] or any other Active Queue Management scheme (AQM) all eventually become oscillatory and prone to instability. In simulation, if iterated for several times without any alteration in RED and network parameters; certain fluctuation is noticed for several TCP variants. Thereby, confirming the unsteadiness of RED queuing with the rise in the number of High-bandwidth-delay products in the network. Table 5, reveals some of such aberrations.

Table 5. Packet received alongside several Parameter Settings of network.

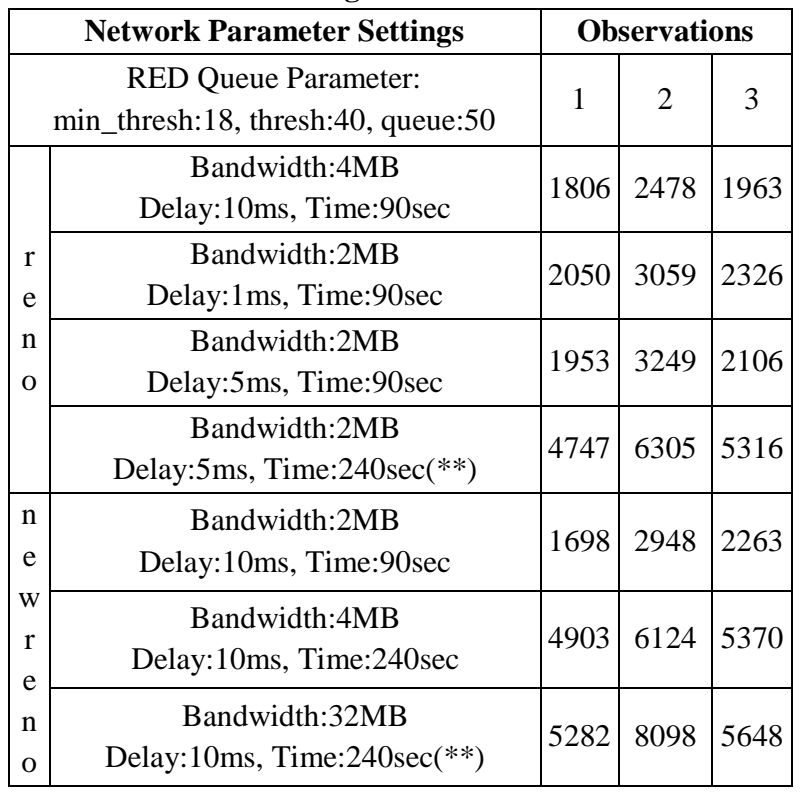

(Table 5. Continued...)

\begin{tabular}{|c|c|c|c|c|}
\hline \multicolumn{2}{|r|}{ Network Parameter Settings } & \multicolumn{3}{|c|}{ Observations } \\
\hline \multirow{3}{*}{$\begin{array}{l}\mathrm{v} \\
\mathrm{e} \\
\mathrm{g} \\
\mathrm{a} \\
\mathrm{s}\end{array}$} & $\begin{array}{c}\text { Bandwidth:32MB } \\
\text { Delay:10ms, Time:90sec }\end{array}$ & 1740 & 2359 & 1935 \\
\hline & $\begin{array}{c}\text { Bandwidth:2MB } \\
\text { Delay:5ms, Time:90sec }\end{array}$ & 1315 & 2197 & 1800 \\
\hline & $\begin{array}{c}\text { Bandwidth:2MB } \\
\text { Delay:100ms, Time:240sec }(* *)\end{array}$ & 4275 & 5607 & 4801 \\
\hline \multirow{6}{*}{$\begin{array}{l}\mathrm{f} \\
\mathrm{a} \\
\mathrm{c} \\
\mathrm{k} \\
1\end{array}$} & $\begin{array}{c}\text { Bandwidth:32MB } \\
\text { Delay:10ms, Time:90sec }\end{array}$ & 1847 & 2612 & 1916 \\
\hline & $\begin{array}{c}\text { Bandwidth: } 2 \mathrm{MB} \\
\text { Delay: } 10 \mathrm{~ms} \text {, Time: } 240 \mathrm{sec}\end{array}$ & 5355 & 5061 & 6297 \\
\hline & $\begin{array}{c}\text { Bandwidth:32MB } \\
\text { Delay:10ms, Time:240sec(**) }\end{array}$ & 5655 & 4947 & 6908 \\
\hline & $\begin{array}{c}\text { Bandwidth:2MB } \\
\text { Delay:5ms, Time:90sec }\end{array}$ & 1767 & 1540 & 2790 \\
\hline & $\begin{array}{c}\text { Bandwidth:2MB } \\
\text { Delay:1ms, Time:240sec }\end{array}$ & 5121 & 6840 & 5993 \\
\hline & $\begin{array}{c}\text { Bandwidth:2MB } \\
\text { Delay:20ms, Time:240sec }\end{array}$ & 5954 & 6835 & 5121 \\
\hline \multirow{4}{*}{$\begin{array}{l}\mathrm{s} \\
\mathrm{c} \\
\mathrm{k} \\
1\end{array}$} & $\begin{array}{c}\text { Bandwidth:2MB } \\
\text { Delay:10ms, Time: } 90 \mathrm{sec}\end{array}$ & 2275 & 2314 & 3250 \\
\hline & $\begin{array}{c}\text { Bandwidth:8MB } \\
\text { Delay:10ms, Time: } 90 \mathrm{sec}\end{array}$ & 1653 & 1849 & 2335 \\
\hline & $\begin{array}{c}\text { Bandwidth:2MB } \\
\text { Delay:10ms, Time: } 240 \sec (* *)\end{array}$ & 6624 & 5188 & 5534 \\
\hline & $\begin{array}{c}\text { Bandwidth:2MB } \\
\text { Delay:1 ms, Time: } 240 \mathrm{sec}\end{array}$ & 4950 & 5641 & 6232 \\
\hline
\end{tabular}

(**) marked observations of 240s simulation are portrayed in the graph of Figure 5 (An aberrant observation/Variant).

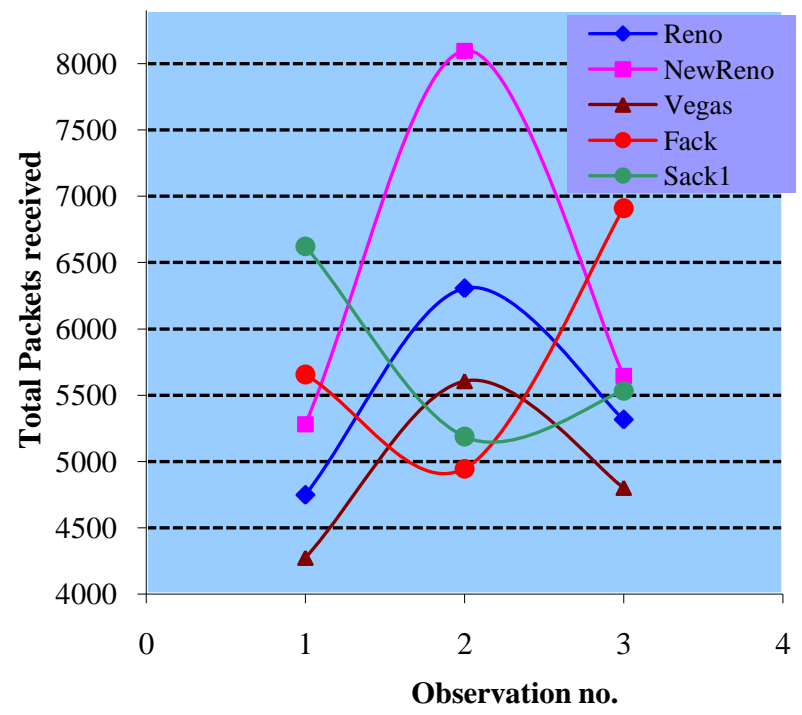

Figure 5.Graph of total packets received for various TCP Variants under the condition of unsteadiness for $240 \mathrm{~s}$. 
As depicted in the Figure 5, we discern easily, performances of RED queuing in case of high-bandwidth-delay networks is undulating and unsteady to a great extent.

\section{CONCLUSION}

For the Internet to flourish incessantly its congestion control mechanism must remain efficient and effective as the network gestates. Our extensive simulations illustrate that some of the TCP Variants maintains good utilization and steadiness over a band of bandwidths or a scale of delays. However, RED is understood to sustain a network steady to a definite limit in network parameter's settings, in addition fails to deal with network extremes. To deal with both steady-state and dynamic environments (with web-like traffic and with impulse loads), a new mechanism XCP is proposed that explicitly deals all sort of congestion problems even for high-bandwidth-delay networks maintaining stability and implying fairness in a better manner at all aspect than any other queuing mechanism does.

\section{REFERENCES}

[1] S. FLOYD and V. JACOBSON: 'Random Early Detection gateways for congestion avoidance'. IEEE/ACM Transactions on Networking, 1993, 1 (4), pp. 397-413.

[2] S. Floyd and V. Jacobson. The synchronization of periodic routing messages. IEEE/ACM Transactions on Networking, 2(2):122\{136, April 1994.

[3] C.V. HOLLOT, VISHAL MISRA, DON TOWSLEY AND WEI-BO GONG: A Control Theoretic Analysis of RED. IEEE INFOCOM 2001

[4] YIGUANG HONG, YONGHAN CAO, HAIRONG SUN, KISHOR S. TRIVEDI: 'RED parameters and Performance of TCP Connections'. In part by ARO-MURI "Mathematics of Failures in Complex Systems".

[5] DINA KATABI, MARK HANDLEY, CHARLIE ROHRS: Congestion Control for High Bandwidth-Delay Product.

[6] NS, The network simulator-ns-2.27. URL:http://www.isi.edu/nsnam/ns

[7] Tanja Lang, (2002), "Evaluation of different TCP versions in non-wireline environments", The University of South Australia, Institute for Telecommunications Research.

[8] "The ns Manual", The VINT Project, A Collaboration between researchers at UC Berkeley, LBL, USC/ISI, and Xerox PARC.

[9] Merida, Venezuela and ESSI, Sophia-Antipolis (2003), "NS Simulator for beginners", Lecture notes, 2003-2004, Univ. de Los Andes, France.

[10] L. Zhang, S. Shenker, and D.D. Clark, Observations on the dynamics of congestion control algorithm: the effects of two way traffic, Proceedings of the Conference on Communications Architecture \&Protocols, 133-147, Zurich, Switzerland, 1991.

[11] R. Mahajan, S. Floyd, and D. Wetherall, Controlling High Bandwidth Flows at the Congested Router, Proceedings of the Ninth International Conference on Network Protocols, 192, November 11-14, 2001.

[12] S. Floyd, J. Mahdavi, M. Mathis, and M. Podolsky, An Extension to the Selective Acknowledgement (SACK) Option for TCP, RFC Editor, 2000.

[13] K. Xu, and N. Ansari, Stability and fairness of rate estimation based AIAD congestion control in TCP, IEEE Communications Letters, 9, 378-380, 2005.

[14] Information Science Institute, Transmission Control Protocol, in: RFC793, 1981.

[15] S. Floyd. RED: Discussions of setting parameters. http://www.aciri.org/floyd/REDparameters.txt, 1997.

[16] S. H. Low, F. Paganini, J. Wang, S. Adlakha, and J. C. Doyle. Dynamics of tcp/aqm and a scalable control. In Proc. of IEEE INFOCOM, June 2002.

[17] S. Athuraliya, V. H. Li, S. H. Low, and Q. Yin. Rem: Active queue management. IEEE Network, 2001

\section{Authors Profile:}

Mohammad Abu Obaida ${ }^{1}$ obtained his Bacholor of Science in Engineering degree from Department of Computer Science and Engineering (CSE), Dhaka University of Engineering \& Technology (DUET), Gazipur, Bangladesh in 2011. At present he is performing extensive research on networks and cryptography. His key research interest includes Cryptography, Network and Web Security, Wireless networking, Software Architecture, Machine Vision, Artificial Intelligence, Protocol analysis and Algorithm design.

Md. Sanaullah Miah achieved his B. Sc. in Engineering degree from Department of Computer Science and Engineering (CSE), DUET, Bangladesh in 2011. Being enthusiastic about mobility management in IPV6, he is currently performing extensive research on the field. Artificial Intelligence, Wireless networking, Cyber Security and Software Engineering are the major research interests to him.

Md. Abu Horaira who serves as a Software engineer to DataSoft Systems Bangladesh Limited, completed Bachelor of Science in Engineering degree from Department of CSE, DUET, Gazipur, Bangladesh in 2009. Pattern Recognition, Artificial Intelligence, System Architecture, Neural and Web Networks are the key subject of interest to him. In addition, Horaira et. al. developed a complete OCR for any local language. 